preprandial values on day 15 (mean[SD]: from 66\%[11] to 64\% [11], $\mathrm{p}=0.091)$.

Conclusion Our results suggest that in preterm infants during their first 36 days of life, cerebral perfusion does not decrease the first hour after feeding. One might reason that most preterm infants may yet be able to regulate cerebral perfusion, or postprandial intestinal perfusion may not increase at all.

\section{PS-190 THE EFFECT OF ENTERAL FEEDING ON REGIONAL INTESTINAL OXYGEN SATURATION IN PRETERM BORN INFANTS}

${ }^{1}$ SJ Kuik, ${ }^{1} \mathrm{TE}$ Schat, ${ }^{2} \mathrm{AGJFvan}$ Zoonen, ${ }^{1} \mathrm{AF}$ Bos, ${ }^{1} \mathrm{KNJ}$ Avan Braeckel, ${ }^{2} \mathrm{JBF}$ Hulscher, ${ }^{1} \mathrm{EMW}$ Kooi. 'Neonatology, Beatrix Children's Hospital/University Medical Center Groningen, Groningen, Netherlands; ${ }^{2}$ Pediatric Surgery, University Medical Center Groningen, Groningen, Netherlands

\subsection{6/archdischild-2014-307384.487}

Background Term born infants demonstrate an increase in intestinal perfusion after receiving enteral feeding (postprandial intestinal hyperemia). It remains unclear whether enteral feeding influences intestinal perfusion in preterm infants.

Aim To assess the effect of enteral feeding on intestinal perfusion in preterm infants.

Methods This study was part of a larger prospective cohort study. We used near-infrared spectroscopy to measure regional intestinal oxygen saturation $\left(\mathrm{r}_{\text {int }} \mathrm{SO}_{2}\right)$, which is indicative for intestinal perfusion. We measured during two hours on postnatal days $2-5,8,15,22,29$, and 36 . Feeding times were manually recorded. We used Multi-level analyses to compare preprandial (baseline) $\mathrm{r}_{\text {int }} \mathrm{SO}_{2}$ values to postprandial $\mathrm{r}_{\text {int }} \mathrm{SO}_{2}$ values, both $10-$ $30 \mathrm{~min}$ and 30-60 min after feeding.

Results We included 29 preterm infants with a median GA of $28+1 / 7$ (range $25+1 / 7-30+4 / 7$ ) weeks, and a median birth weight of 1025 (range 580-1495) grams. Compared to preprandial values, we only found decreased postprandial $\mathrm{r}_{\text {int }} \mathrm{SO}_{2}$ values 30-60 min after feeding (mean[SD]: from 47\%[22] to 41\%[19], $\mathrm{p}=0.007$ ) on day 5. We observed increased $\mathrm{r}_{\text {int }} \mathrm{SO}_{2}$ values $10-$ 30 min after feeding compared to preprandial values on day 29 (mean[SD]: from 51\%[14] to 42\%[14], $\mathrm{p}=0.005$ ).

Conclusion Our results suggest that in preterm infants during their first three weeks of life, intestinal oxygen saturation does not increase, and sometimes even decreases, the first hour after feeding. One might speculate that preterm infants are not yet able to increase intestinal perfusion rates after feeding, an increase which might be necessary to meet metabolic demands.

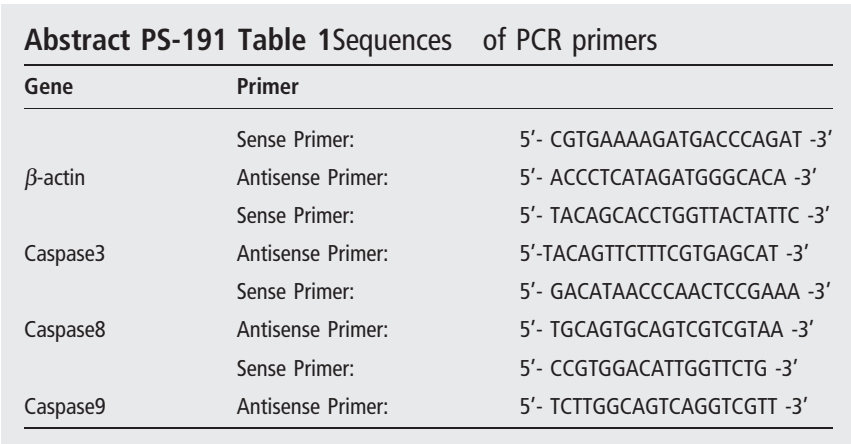
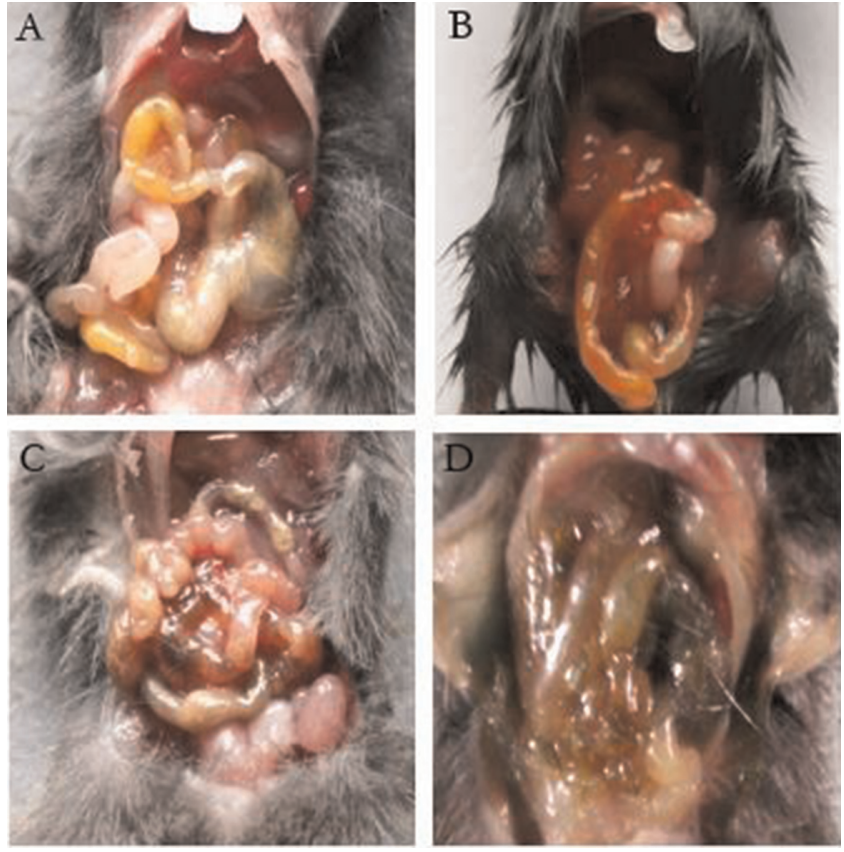

Abstract PS-191 Figure 1 TLR4-deficient mice were protected from the development of NEC. Abdominal anatomy of mice in the different groups. Wild type C57BL/10j mice (A) or TLR4-deficient C57BL/10SCNJ mice $(C)$ were served as breast-fed controls. NEC was induced in newborn Wild type C57BL/10j mice (B) or TLR4-deficient C57BL/10SCNJ mice (D).

\section{PS-191 TOLL LIKE RECPTOR-4 SIGNALLING MEDIATED APOPTOSIS IN NECROTIZING ENTEROCOLITIS VIA CASPASES ACTIVATION}

${ }^{1} \mathrm{Y}$ Zhou, ${ }^{1} \mathrm{~B}$ Liu, ${ }^{2} \mathrm{~B}$ Zhou, ${ }^{2} \mathrm{KL}$ Chen, ${ }^{1} \mathrm{GZ}$ Yang, ${ }^{1} \mathrm{Y}$ Wang, ${ }^{1} \mathrm{LP}$ Yao, ${ }^{1} \mathrm{Y}$ Li. ${ }^{1}$ Department of Pediatric Surgery, West China Hospital of Sichuan University, Chengdu, China; ' Institute of Digestive Surgery State Key Laboratory of Biotherapy, West China Hospital of Sichuan University, Chengdu, China

\subsection{6/archdischild-2014-307384.488}

Background and aim The importance of Toll-like receptor-4 (TLR4) signalling in necrotizing enterocolitis (NEC) is well-documented, but the potential mechanisms that regulate enterocyte apoptosis remain unclear. We investigated the role of apoptosis factors-Caspases in NEC and its pathway (endogenous and/or exogenous).

Methods TLR4-deficient C57BL/10ScNJ mice and Lentivirusmediated stable TLR4-silent cell line (IEC-6) were used. NEC was induced by formula gavage, cold, hypoxia, combined with LPS in vivo or LPS stimulation in vitro. NEC severity was evaluated by histology. Enterocyte apoptosis was evaluated by TUNEL or Annexin analysis. The expression of TLR4, Caspases8, Caspase9, and Caspase 3 were detected by qRT-PCR and western blot. Inflammatory factors including TNF- $\alpha$, IL-6, IL-10 and IL-2 were examined by Luminex.

Results In TLR4-deficient mice, the severity of NEC was reduced, the expression of caspase 8 was decreased, caspase 9 was increased, and Caspase 3 did not change significantly. In TLR4silent IEC-6 cell line, expression of caspase 3 was decreased and apoptosis rate were significantly reduced. Cytokine level of TNF- $\alpha$ and IL-2 were decreased.

Conclusion TLR4 induced apoptosis plays a critical role in the pathogenesis in NEC. Defect of TLR4 inhibits enterocytes from apoptosis in NEC, predominantly through Caspase8-mediated apoptosis pathway. 

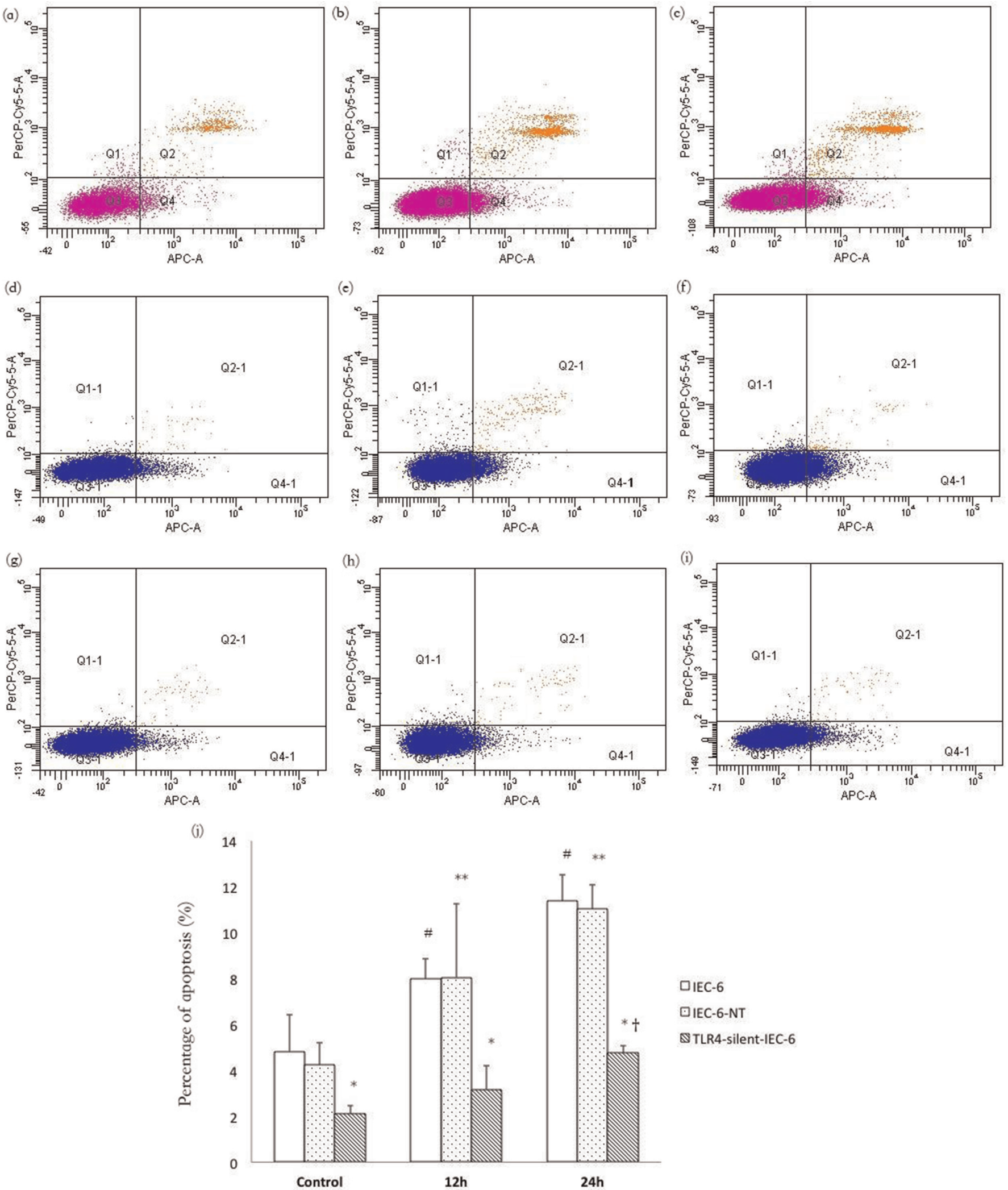

口IEC-6

๑IEC-6-NT

$\mathbb{Q}$ TLR4-silent-IEC-6

Abstract PS-191 Figure 2 FCM detection of apoptosis after stimulation of LPS (50 $\mu \mathrm{g} / \mathrm{ml})$ at different times in vitro. (a) IEC-WT control group; (b) $12 \mathrm{~h}$ after stimulation with LPS in IEC-WT group; (c) $24 \mathrm{~h}$ after stimulation with LPS in IEC-WT group; (d) IEC-6 transfected with vector only (Non Target, NT) control group; (e) $12 \mathrm{~h}$ after stimulation with LPS in IEC-NT group; (f) $24 \mathrm{~h}$ after stimulation with LPS in IEC-NT group; (g) TLR4-silentIEC-6 control group; (h) $12 \mathrm{~h}$ after stimulation with LPS in TLR4-silent-IEC-6 group; (i) $24 \mathrm{~h}$ after stimulation with LPS in TLR4-silent-IEC-6 group; The Q4 quadrant (annexin V+/7-AAD-) indicate the percentage of apoptosis. (j) Quantification of apoptosis percentage determined by a blinded observer. ${ }^{*} \mathrm{P}<0.05$ by ANOVA compared with IEC-6-WT and IEC-6-NT group at the same time point, \#P $<0.05$ by ANOVA compared with control within IEC6 -WT group, ${ }^{* *} \mathrm{P}<0.05$ by ANOVA compared with control within IEC-6-NT group, $+\mathrm{P}<0.05$ by ANOVA compared with control within TLR4-silentIEC-6 group. Representative data from four separate experiments with three times per group. 